\title{
Weighted Marking, Clique Structure and Node- Weighted Centrality to Predict Distribution Centre's Location in a Supply Chain Management
}

\author{
Akanmu, Amidu A. G., \\ School of Computing, \\ University of Kent, Medway, \\ ME4 4AG, Kent
}

\author{
Wang, Frank Z., \\ School of Computing, \\ University of Kent, Canterbury, \\ Kent
}

\author{
Yamoah, Fred A., \\ School of Business, \\ University of Kent, Medway, \\ ME4 4AG, Kent
}

\begin{abstract}
Despite the importance attached to the weights or strengths on the edges of a graph, a graph is only complete if it has both the combinations of nodes and edges. As such, this paper brings to bare the fact that the node-weight of a graph is also a critical factor to consider in any graph/network's evaluation, rather than the link-weight alone as commonly considered. In fact, the combination of the weights on both the nodes and edges as well as the number of ties together contribute effectively to the measure of centrality for an entire graph or network, thereby clearly showing more information. Two methods which take into consideration both the link-weights and node-weights of graphs (the Weighted Marking method of prediction of location and the Clique/Node-Weighted centrality measures) are considered, and the result from the case studies shows that the clique/node-weighted centrality measures give an accuracy of $18 \%$ more than the weighted marking method, in the prediction of Distribution Centre location of the Supply Chain Management.
\end{abstract}

Keywords-Centrality measures; Graph; Network; Clique

\section{INTRODUCTION}

The formal theory of social network analysis encompasses centrality measures, and these are to be employed in this research that dwells on the mergers of weights (link-weights and node weights) to evaluate network topologies and make a prediction. The strength attached to the nodes also called the node-weights represents a certain attribute of a particular node (e.g. population of a city), and the same goes for the strength attached to edges (e.g. distance between cities).[1]

According to [2], in their study of weighted networks, they carried out statistical analysis of complex networks whose edges have assigned a given weight (the flow or the intensity), and such according to them can generally be described in terms of weighted graphs and more so that a more complete view of complex networks is provided by the study of the interactions defining the links of those systems. Although, [3], [4], [2], [5] have only emphasized on the attachment of weights to the edges and not to the nodes in their various studies, [6] and [7] have considered both the weights on the edges and also the number of edges attached to a particular node. This work however concerns itself with both nodes and edges while considering the degree, eigenvector, betweenness and closeness centralities.
The importance of location of distribution centre is echoed by [8], when they said "Moreover, the advantage of an optimal location for distribution centre is not only to reduce transportation costs, but also to improve business performance, increase competitiveness and profitability".

Although, [9] indicated that "Rarely do members of a group have direct ties with each and every member.", the case studies we focused on are the road links(edges) to shops coupled with the node weights(sales values of each shop) and this is such that every shop has a road link to another shop thereby forming a clique.

\section{Link-Weighted Centrality}

The equation (1) below represents the weighted degree centrality with respect to the edges or links.

$S_{p}=C_{D}^{W}(p)=\frac{\sum_{q}^{N} w_{p q}}{n-1}$

where $\mathrm{p}$ is the focal node ; $\mathrm{q}=$ adjacent node ; $\mathrm{w}=$ weight attached to the edge ; and $n=$ total number of nodes in the graph.

The above argument is now extended to the weighted centrality of the four measures, i.e. Degree, Closeness, Betweenness and the Eigenvector. The degree centrality of any node $S$ taking cognisance of the strength of the incident edges is herein defined as the weighted degree centrality of node $s$ and is represented in normalised form as

$$
C_{D}^{W}(s)=\frac{t^{n} w_{s t}}{n \quad 1}
$$

where $w_{s t}$ is the sum of the weights of edges connected to the particular source node $s$ and $t$ represents a particular target node. In the same vein, the weighted closeness centrality, $c_{c}^{w}$ $(s)$ is also represented by

$C_{C}^{W}(s)=\frac{n 1}{d_{w}(s, t)}$ 
where $d_{w}(s, t)$ is the weight of geodesic paths between $s$ and $\mathrm{t}$, while the weighted betweenness centrality is

$$
C_{B}^{W}(v)=\underbrace{}_{\substack{s, v t \\
s t}} \frac{{ }^{w}(v) /}{\left(\begin{array}{ll}
n & 1
\end{array}\right)\left(\begin{array}{ll}
n & 2
\end{array}\right)}
$$

where $s_{s t}$ is the number of the shortest geodesic paths from $\mathrm{s}$ to $\mathrm{t},{ }_{s t}(\mathrm{v})$ is the number of the shortest geodesic paths from $\mathrm{s}$ to $\mathrm{t}$ that pass through node $\mathrm{v}$ and $\mathrm{w}$ is the assigned weights to the ties. Similarly, the weighted eigenvector centrality could be seen as

$$
\lambda \mathbf{x}=\mathbf{A}^{\mathbf{w}} \mathbf{x}
$$

where $\mathbf{A}^{\mathbf{w}}$ is a square matrix of the weights on the edges of $\mathbf{A}$ and $\mathbf{x}$ is an eigenvector of $\mathbf{A}$.

A tuning parameter $\alpha$ was introduced to determine the relative importance of the number of ties compared to the weights on the ties by [7]. Equation (6) below thereby represents the product of degree of a focal node and the average weight to these nodes as adjusted by the introduced tuning parameter. So, for weighted degree centrality at $\alpha$ we have:

$c_{d}^{w \alpha}(p)=k_{p} \times\left(\frac{s_{p}}{k_{p}}\right)^{\alpha}=k_{p}^{(1-\alpha)} \times s_{p}^{\alpha}$

where $\mathrm{k}_{\mathrm{p}}=$ degree of nodes

$\mathrm{S}_{\mathrm{p}}=c_{D}^{w}(\mathrm{p})$ as defined in (1) above, and $\alpha$ is $\geq 0$

So for weighted closeness centrality at $\alpha$ we have

$$
c_{c}^{w}(i)=k_{i} \quad\left(\frac{s_{i}}{k_{i}}\right)=k_{i}^{(1} \quad s_{i}
$$

where $\mathrm{k}_{\mathrm{i}}=$ degree of nodes and $s_{i}=c_{C}^{w}(s)$ is as defined in (3) above, $\alpha$ is $\geq 0$, and similarly for the degree centrality; betweenness centrality and eigenvector centrality.

\section{Weights On Nodes}

In the supply chain management (SCM), the node-weights could be any of the volume of sales, cost of storage or turnover at a depot/store, while the edges will be the distance between each depot and a proposed distribution centre (DC). TESCO shops of different counties are used as case studies here.

For the SCM, since the shops sampled are maximally connected, the advantage of the clique structure was exploited to map out different clique of shops and thereby making the most central node of the chosen clique to be representative of that clique for the purpose of prediction of a proposed DC.

\section{Node-Weight Modified Centrality Measure}

From (6), when $\alpha=0$ only the degree of nodes will be measured and if $\alpha=1$ only the weights on the ties are measured.
In view of this only the cases whereby $\alpha$ is less than 1 or greater than 1 shall be considered, specifically cases of $\alpha$ $=0.25 ; 0.5 ; 0.75 ; 1.25 ; 1.5$ and 1.75

A tuning parameter $\beta$ was introduced by [1] to take care of the weightedness on the nodes, although the tuning parameter $\alpha$ was applied to the degree/strength of the edges. The newly evolved equation by way of introduction of a tuning parameter $\beta$ will now be the product of degree of a focal node, the average weight to these nodes as adjusted by the newly introduced tuning parameter $\beta$ and the weight accorded to each node. So, for weighted degree centrality at $\alpha$ and $\beta$ we shall now have

$$
\left.c_{d}^{w} \quad(i)=k_{i} \quad\left(\frac{s_{i}}{k_{i}}\right)=k_{i}^{(1}\right) \quad s_{i} \quad z_{i}
$$

where $\mathrm{k}_{\mathrm{i}}=$ degree of nodes

$s_{i}=c_{D}^{w}(s)$ as defined

$\mathrm{Z}_{\mathrm{i}}=$ weight of nodes, where $\alpha \geq 0 ;\{\beta \in Z:-1 \leq \beta \leq 1\}$

The choice of value of $\beta$ depends on what effect the weight is having on the new centrality measure, if for instance the weight is having a positive effect (e.g. profit) the positive value of $\beta$ is employed otherwise the negative value(e.g. loss) shall be used in our calculation.

The next sections are organised as follow, section II discusses the two methods Weighted Marking Method and Clique Structure/Node-Weight Modulated Centrality Measure as applicable to the supply chain management. Section III explains the implementation of the exercise with such tools as UCINET, tnet and Excel while section IV shows the predictions of the new distribution centres. Section V concludes with the discussion of the results.

\section{WeIGHTED MARKING METHOD AND CliQUE STRUCTURE/NODE-WEIGHT MODULATED CENTRALITY MEASURES APPLIED TO SUPPLY CHAIN MANAGEMENT}

\section{A. Weighted Marking Method}

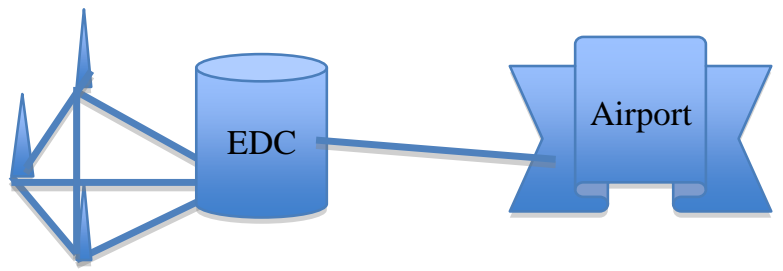

Fig. 1. Figure showing schematic diagram of Weighted Marking Method, with cones as shops \& EDC as existing DC

Three main stages were proposed by [8] in choosing a location for DC using the Weighted Marking Method (WMM):

Stage1 - Identification of a general geographical area for DC based on the principle of centre of gravity while considering socio-economic factors. For the Scotland region in our case study, Glasgow and Edinburgh are considered as being the most populated and with tendencies for more economic activities. 
Stage 2 - Identification of alternative locations of DC, these are the shops (cones) as in fig.1 whereby EDC is the existing DC. The considered criteria for the cities in stage1 are: Criteria1 - C1 (proximity to customer bases); C2(Expansion capability); $\mathrm{C} 3$ (percentage of unemployment [to measure availability of labour force]) and C4(Average Income of residents[to measure standard of living]).

Stage3 - Selection of specific sites among the alternative locations in Stage2 using quantitative approach after having set a certain treshold (e.g. Composite functions greater than or equal to 5), i.e. the composite point for each node is calculated using the formula below:

Composite Point $=\sum_{1}^{4}($ Point related to each criteria * weighting factor of that criteria)

Thereafter the minimum from the products of Sales Volume and Distance is chosen as in (10) below

$$
\operatorname{Min}\{\mathrm{VD}\}=\min \{\text { Volume of Sales*Distance }\}
$$

Applying the technique of [8], the result of Table1 was obtained:

From above, node 79 is the winner and its distance from the existing DC is 14.1 units, therefore the error of prediction is $14.1 / 60 * 100=23.5 \%$, which gives an accuracy of $76.5 \%$.

The network coverage of an existing distribution centre (DC) located at Scotland was investigated and the retail outlets or shops are considered as nodes with the value of sales taken to be the weights on the nodes while distances between nodes are regarded as the weights on the edges. For our sample a 30 miles radius coverage of shops from the existing DC was taken and this makes 63nodes all connected by distances (see fig.2 below). The nearest DC to this existing one is some 171 miles away, so our coverage for this purpose is of 60miles diameter, although this could be extended in future. Out of the community of 63 shops, the Central and Lothian Counties accommodated 43 of these shops while Glasgow city and Edinburgh have 30 of these. The existing DC at Livingston is actually situated in-between these two cities. The clique of shops within Glasgow and Edinburgh were examined and the most central from the two cliques were considered for the prediction of the new DC.

\section{B. Clique Structure/Node-Weight Modulated Centrality Measures}

The first case study was the region of Scotland and the second was for the region of Northern Ireland. As depicted in fig.2. below, the clique of a graph is considered from among which the most central of the nodes is taken to be representative of that clique, which in turn is considered for the prediction test along with the other cliques. From the NodeWeighted Centrality Measure, the two nodes 5 and 22 are the most central in terms of the node-weightedness, thereby representing the cliques of Glasgow and Edinburgh respectively.

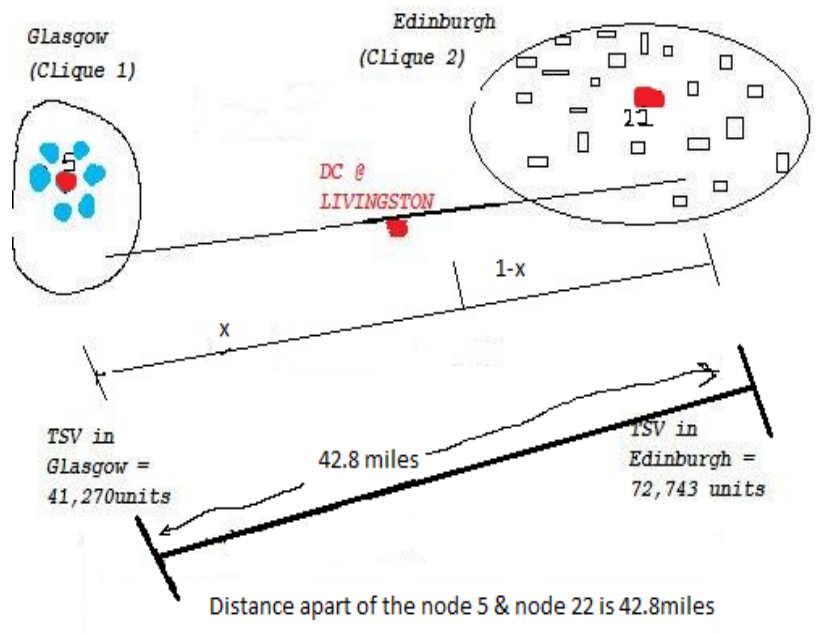

TSV = TOTAL SALES VALUE AT EACH NODE

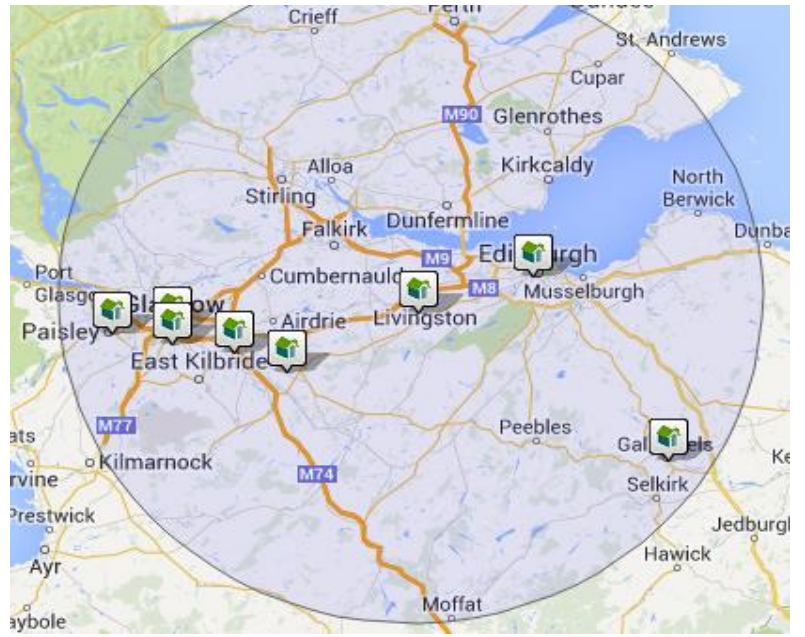

Fig. 2. Figure showing the coverage of the 30miles radius of Scotland, cliques at Glasgow \& Edinburgh ( Source : www.rightmove.co.uk ) 

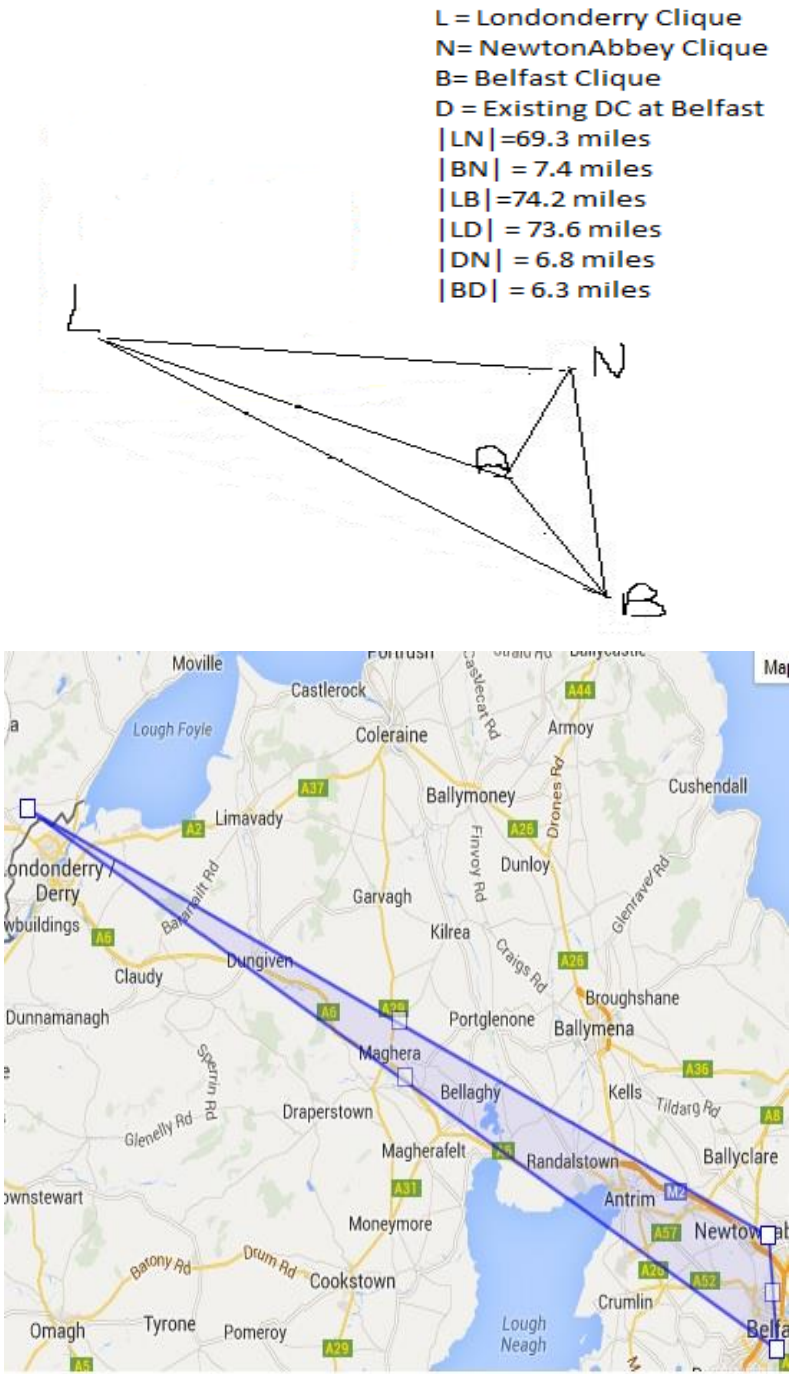

Fig. 3. Figure showing the cliques at Northern Ireland cities (Londonderry, NewtonAbbey \& Belfast)

Tables II \& III below show the respective results that were obtained when the Node-Weight Modulated Centrality Measures are applied to the 7 nodes of Glasgow and 23 nodes of Edinburgh from the supply chain management dataset.

\section{IMPLEMENTATION}

The initial dataset of the distances between the 30 sales outlets of Glasgow and Edinburgh's cliques were presented as a $7 \times 7$ square matrix and $23 \times 23$ square matrix respectively, these were obtained from the UCINET and tnet software, saved in Excel format and later imported into UCINET for the purpose of centralities calculations, see fig. 4 below.

The results came out as text files listing the different columns for each centrality measure, and for the purpose of calculations of the node-weight modulated centrality, the values from the text files were exported into Excel where a column was created for the weights on the nodes. Tables II \& III below depict the node-weighted centrality measures at different values of alpha (in terms of degree, closeness, eigenvector and betweenness).

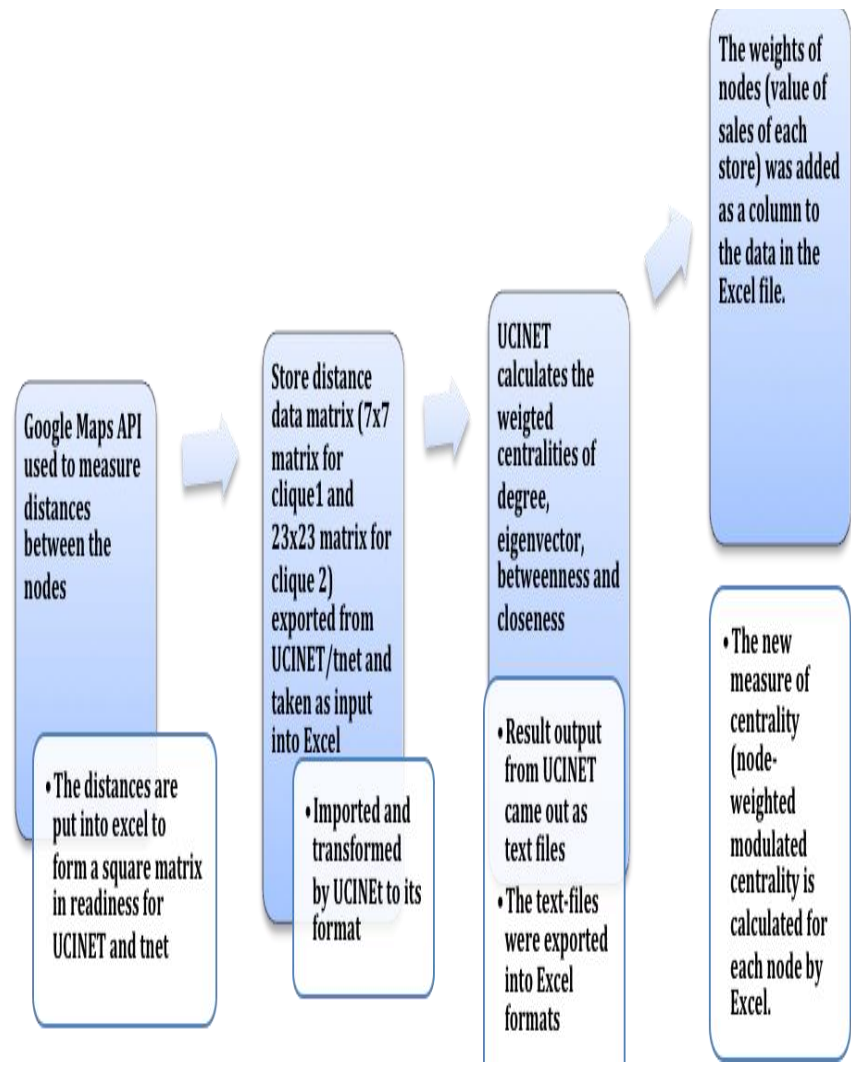

Fig. 4. Figure showing the implementation of node-weighted centrality measure to the cliques of SCM

The radius of coverage according to fig. 1 is 30miles, accordingly the farthest possible distance apart of any two nodes will be the diameter of such circle which is 60 miles. This was used for the calculation of ratio of distances from any node to that of farthest distance apart. The percentage error of prediction is therefore calculated by multiplying this ratio by 100 and from this emerges the percentage accuracy.

\section{Clique/Node-Weighted Measure APplied to the SUPPLY CHAIN MANAGEMENT (SCM)}

$x$ is the proportional distance to the proposed Distribution Centre. TSV $=$ Total Sales Values

The driving distance between node 5 (representing Glasgow clique) and node 22 (representing Edinburgh clique) is 42.8 miles.

$1-\mathrm{x} / \mathrm{x}=41270 / 72743$

$\mathrm{x}=0.36$ (i.e. $36 \%$ of 42.8 ) which is 13.1 miles

If $\mathrm{x}$ is some 13.1 miles away from the highest sales valued node 22 (Edinburgh), and the existing DC is 15.4 miles away from the same node 22, the difference of the predicted DC will be 2.3miles away from the existing DC, hence

The error rate of the predicted $\mathrm{DC}=(2.3 / 42.80) \times 100=$ $5.37 \%$ i.e. the percentage accuracy of the prediction $=94.63 \%$ 
With all the results above one is now in a position to predict the most probable (regions with respect to the nodes) that could serve as a distribution center for all other outlets considering their node-weighted centrality and clique structures going by the percentage accuracy of the prediction.

Similar argument is also extended to some 51 shops at the Northern Ireland whereby three cliques are considered, that is the cliques at Belfast (14 shops); Londonderry (three shops) and NewtonAbbey (four shops). See fig. 3 above, whereby the centre of mass of the triangle was considered to be the predicted Distribution Centre, while the angles of the nodes are calculated using the cosine rule, see fig. 5 below:

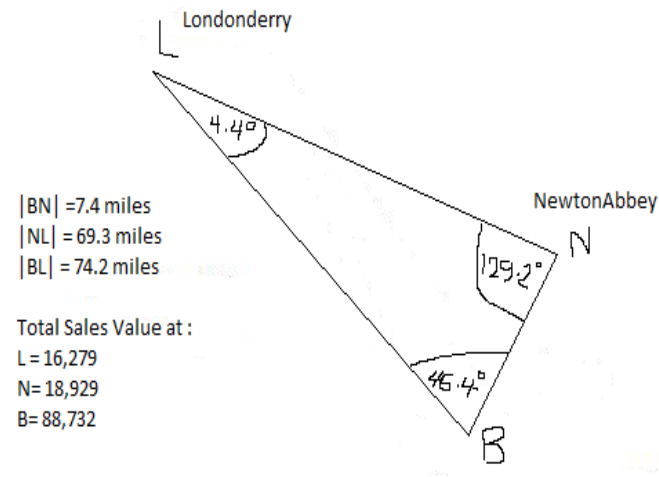

Belfast

Fig. 5. Figure showing the angles of triangle BNL, distances apart of the nodes and sales values of each clique

Since only the distance between the shops are available, from the calculated angles the co-ordinates were arrived at. See fig.6.

From the figure above, $\mathrm{B}(0,0)$ indicates origin whereby $\mathrm{x}_{1}=$ 0 and $\mathrm{y}_{1}=0$

$$
\begin{aligned}
a_{1} & =74.2 \operatorname{Cos} 46.4=51.17 \\
h & =74.2 \operatorname{Sin} 46.4=53.73
\end{aligned}
$$

The co-ordinate of the centre of mass for the triangle $\mathrm{BNL}$ is calculated using facts from fig.6. above, whereby $\mathrm{x}_{1}=0 ; \mathrm{x}_{2}=$ $7.4 ; \mathrm{x}_{3}=51.17 ; \mathrm{y}_{1}=0 ; \mathrm{y}_{2}=0 ;$ and $\mathrm{y}_{3}=53.73$

Total sales value at clique $\mathrm{B}\left(\mathrm{w}_{1}=88732\right)$; at clique $\mathrm{N}\left(\mathrm{w}_{2}\right.$ $=18929)$ and at clique $\mathrm{L}\left(\mathrm{w}_{3}=16279\right)$.

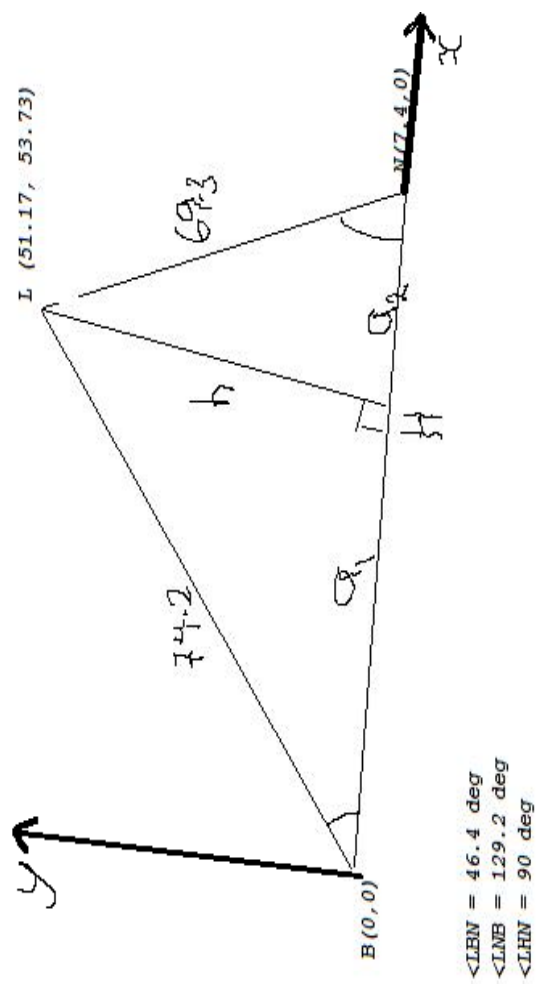

Fig. 6. Figure showing the co-ordinates of the triangle

Hence, for the predicted DC (the centre of mass), the coordinates are

$x_{c m}=\frac{1}{n} \frac{\sum_{i=1}^{n} m_{i} x_{i}}{M}$

$y_{c m}=\frac{1}{n} \frac{\sum_{i=1}^{n} m_{i} y_{i}}{M}$

where $M=\sum_{i=1}^{n} m_{i} \quad$ (the total weights on the nodes) and $\mathrm{n}=$ number of nodes/vertices. 
Substituting in the values from fig.6 above, equations (ii) and (iii) become respectively,

$$
\begin{gathered}
x_{\mathrm{cm}}=\{[0 \times 88732] /[88732+18929+16279]+[7.4 \mathrm{x} \\
18929] /[88732+18929+16279]+[51.17 \mathrm{x} \\
16279] /[88732+18929+16279]\} / 3
\end{gathered}
$$

therefore, $x_{\mathrm{cm}}=2.62$. Similarly, $y_{\mathrm{cm}}=2.35$

So, the predicted DC has co-ordinates $(2.62,2.35)$, hence the distance from clique $\mathrm{B}$ as shown in fig.7.

$$
\mathrm{BP}=\left\{2.35^{2}+2.62^{2}\right)^{1 / 2}=3.52
$$

From fig. 3 above, the existing DC is 6.3 miles to the clique at Belfast, so the error is $6.3-3.52=2.78$, that is, the percentage error is $(2.78 / 73.6 \times 100)$, the farthest distance apart from the existing DC being 73.6, therefore the percentage accuracy for this prediction is $96.2 \%$.

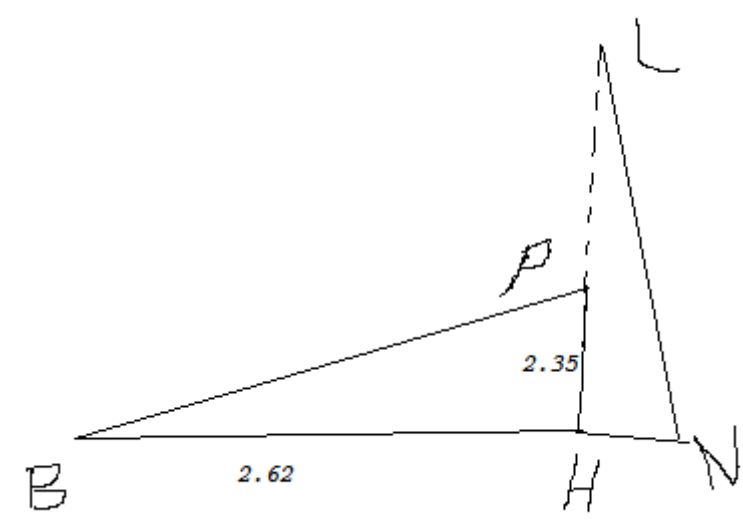

\begin{tabular}{|c|c|c|c|c|c|c|c|c|c|c|c|c|c|c|c|c|}
\hline & & & & $\mathrm{C} 1$ & & $\begin{array}{l}\text { \%AGE } \\
\text { of } \mathrm{Cl}\end{array}$ & & $\begin{array}{l}\text { \%AGE } \\
\text { of } \mathrm{C} 2\end{array}$ & $\mathrm{C} 3$ & & $\begin{array}{l}\text { \%AGE } \\
\text { of C3 }\end{array}$ & $\mathrm{C} 4$ & & $\begin{array}{l}\% \text { AGE } \\
\text { of } \mathrm{C} 4\end{array}$ & & \\
\hline $\begin{array}{l}\text { No } \\
\text { de } \\
\text { No }\end{array}$ & $\begin{array}{l}\text { Unit } \\
\text { s } \\
\text { Sold }\end{array}$ & $\begin{array}{c}\text { Store } \\
\text { Format }\end{array}$ & $\begin{array}{l}\text { Town } \\
\text { /City }\end{array}$ & $\begin{array}{c}\text { Distan } \\
\text { ce to } \\
\text { Existi } \\
\text { ng DC }\end{array}$ & $\begin{array}{c}\text { Point of } \\
\text { Evaluati } \\
\text { on }\end{array}$ & $\begin{array}{c}\text { PT X } \\
\text { WT } \\
\text { FACT } \\
\text { OR }\end{array}$ & $\begin{array}{l}\text { Expansi } \\
\text { on } \\
\text { capacity }\end{array}$ & $\begin{array}{c}\text { PT X } \\
\text { WT } \\
\text { FACT } \\
\text { OR }\end{array}$ & $\begin{array}{c}\text { Availabil } \\
\text { ity of } \\
\text { W/Force }\end{array}$ & $\begin{array}{c}\text { Point of } \\
\text { Evaluati } \\
\text { on }\end{array}$ & $\begin{array}{c}\text { PT X } \\
\text { WT } \\
\text { FACT } \\
\text { OR }\end{array}$ & $\begin{array}{c}\text { Standa } \\
\text { rd of } \\
\text { Living }\end{array}$ & $\begin{array}{c}\text { Point of } \\
\text { Evaluati } \\
\text { on }\end{array}$ & $\begin{array}{c}\text { PT X } \\
\text { WT } \\
\text { FACT } \\
\text { OR }\end{array}$ & $\begin{array}{l}\text { Compos } \\
\text { ite Point }\end{array}$ & $\begin{array}{c}\text { PRODU } \\
\text { CT OF } \\
\text { DIST. } \\
\text { TO DC } \\
\text { \&UNITS } \\
\text { SOLD }\end{array}$ \\
\hline 1 & $\begin{array}{r}439 \\
0 \\
\end{array}$ & Extra & $\begin{array}{c}\text { GLASGO } \\
\text { W }\end{array}$ & 29.5 & 1 & 0.25 & 8 & 2 & VH & 9 & 2.25 & 852 & 7 & 1.75 & 6.25 & 129505 \\
\hline 6 & $\begin{array}{r}930 \\
0 \\
\end{array}$ & Extra & $\begin{array}{c}\text { GLASGO } \\
\text { W } \\
\end{array}$ & 29 & 2 & 0.5 & 8 & 2 & VH & 9 & 2.25 & 852 & 7 & 1.75 & 6.5 & 269700 \\
\hline 8 & $\begin{array}{r}107 \\
90 \\
\end{array}$ & Extra & $\begin{array}{c}\text { GLASGO } \\
\mathrm{W} \\
\end{array}$ & 30 & 1 & 0.25 & 8 & 2 & FH & 7 & 1.75 & 860 & 7 & 1.75 & 5.75 & 323700 \\
\hline 55 & $\begin{array}{r}147 \\
80 \\
\end{array}$ & Extra & $\begin{array}{c}\text { EDINBUR } \\
\text { GH }\end{array}$ & 15 & 9 & 2.25 & 8 & 2 & - & $\mathbf{0}$ & 0 & 939 & 9 & 2.25 & 6.5 & 221700 \\
\hline 56 & $\begin{array}{r}111 \\
0 \\
\end{array}$ & Metro & $\begin{array}{c}\text { EDINBUR } \\
\text { GH }\end{array}$ & 20.3 & 6 & 1.5 & 4 & 1 & VL & 3 & 0.75 & 867 & 7 & 1.75 & 5 & 22533 \\
\hline 57 & $\begin{array}{r}185 \\
0 \\
\end{array}$ & Metro & $\begin{array}{c}\text { EDINBUR } \\
\text { GH }\end{array}$ & 17.1 & 7 & 1.75 & 4 & 1 & LST & 1 & 0.25 & 939 & 9 & 2.25 & 5.25 & 31635 \\
\hline 64 & $\begin{array}{r}532 \\
0 \\
\end{array}$ & $\begin{array}{c}\begin{array}{c}\text { Superst } \\
\text { ore }\end{array} \\
\end{array}$ & $\begin{array}{c}\text { EDINBUR } \\
\text { GH }\end{array}$ & 20.3 & 6 & 1.5 & 6 & 1.5 & FH & 7 & 1.75 & 860 & 7 & 1.75 & 6.5 & 107996 \\
\hline 65 & $\begin{array}{r}104 \\
80 \\
\end{array}$ & $\begin{array}{c}\begin{array}{c}\text { Superst } \\
\text { ore }\end{array} \\
\end{array}$ & $\begin{array}{c}\text { EDINBUR } \\
\text { GH }\end{array}$ & 17.9 & 7 & 1.75 & 6 & 1.5 & FH & 7 & 1.75 & 860 & 7 & 1.75 & 6.75 & 187592 \\
\hline 66 & $\begin{array}{r}940 \\
0 \\
\end{array}$ & $\begin{array}{c}\text { Superst } \\
\text { ore }\end{array}$ & $\begin{array}{c}\text { EDINBUR } \\
\text { GH }\end{array}$ & 13.1 & 9 & 2.25 & 6 & 1.5 & VH & 9 & 2.25 & 852 & 7 & 1.75 & 7.75 & 123140 \\
\hline 67 & $\begin{array}{r}615 \\
0 \\
\end{array}$ & $\begin{array}{c}\text { Superst } \\
\text { ore }\end{array}$ & $\begin{array}{c}\text { EDINBUR } \\
\text { GH }\end{array}$ & 21.2 & 5 & 1.25 & 6 & 1.5 & FH & 7 & 1.75 & 860 & 7 & 1.75 & 6.25 & 130380 \\
\hline 71 & 130 & Express & $\begin{array}{c}\text { EDINBUR } \\
\text { GH }\end{array}$ & 19 & 7 & 1.75 & 2 & 0.5 & FH & 7 & 1.75 & 810 & 5 & 1.25 & 5.25 & 2470 \\
\hline 72 & 690 & Express & $\begin{array}{c}\text { EDINBUR } \\
\text { GH }\end{array}$ & 19.4 & 6 & 1.5 & 2 & 0.5 & FH & 7 & 1.75 & 860 & 7 & 1.75 & 5.5 & 13386 \\
\hline 73 & 200 & Express & $\begin{array}{c}\text { EDINBUR } \\
\text { GH }\end{array}$ & 25.8 & 3 & 0.75 & 2 & 0.5 & VH & 9 & 2.25 & 852 & 7 & 1.75 & 5.25 & 5160 \\
\hline 74 & 230 & Express & $\begin{array}{c}\text { EDINBUR } \\
\text { GH }\end{array}$ & 15.8 & 8 & 2 & 2 & 0.5 & VL & 3 & 0.75 & 899 & 7 & 1.75 & 5 & 3634 \\
\hline 75 & 260 & Express & $\begin{array}{c}\text { EDINBUR } \\
\text { GH }\end{array}$ & 17.1 & 7 & 1.75 & 2 & 0.5 & FH & 7 & 1.75 & 860 & 7 & 1.75 & 5.75 & 4446 \\
\hline 77 & 890 & Express & $\begin{array}{c}\text { EDINBUR } \\
\text { GH }\end{array}$ & 22.7 & 5 & 1.25 & 2 & 0.5 & FH & 7 & 1.75 & 860 & 7 & 1.75 & 5.25 & 20203 \\
\hline 78 & 390 & Express & $\begin{array}{c}\text { EDINBUR } \\
\text { GH }\end{array}$ & 17.7 & 7 & 1.75 & 2 & 0.5 & FH & 7 & 1.75 & 860 & 7 & 1.75 & 5.75 & 6903 \\
\hline 79 & 70 & Express & $\begin{array}{c}\text { EDINBUR } \\
\text { GH }\end{array}$ & 14.1 & 9 & 2.25 & 2 & 0.5 & VL & 3 & 0.75 & 860 & 7 & 1.75 & 5.25 & 987 \\
\hline 80 & 320 & Express & $\begin{array}{c}\text { EDINBUR } \\
\text { GH }\end{array}$ & 20.8 & 6 & 1.5 & 2 & 0.5 & FH & 7 & 1.75 & 860 & 7 & 1.75 & 5.5 & 6656 \\
\hline 82 & $\mathbf{5 2 0}$ & Express & $\begin{array}{c}\text { EDINBUR } \\
\text { GH }\end{array}$ & 16.5 & 8 & 2 & 2 & 0.5 & LO & 5 & 1.25 & 860 & 7 & 1.75 & 5.5 & 8580 \\
\hline 84 & 700 & Express & $\begin{array}{c}\text { EDINBUR } \\
\text { GH }\end{array}$ & 21.3 & 5 & 1.25 & 2 & 0.5 & FH & 7 & 1.75 & 860 & 7 & 1.75 & 5.25 & 14910 \\
\hline
\end{tabular}

Fig. 7. figure showing the carved out portion of triangle $\mathrm{BPH}$ from triangle BNL of fig. 6 above. $\mathrm{P}$ is the predicted DC

TABLE I. RESUlts of WeIGHTED MARKING METHOD APPLIED to SCOTLAND DATASETS 
TABLE II. TABle Showing the Node-Weighted Centralities at DifFerent Alpha AND AT Beta=1 For Glasgow (CliQue1)

\begin{tabular}{|c|c|c|c|c|c|c|c|c|c|c|c|c|c|c|c|c|c|c|c|c|c|c|c|c|}
\hline & \multicolumn{6}{|c|}{ NODE-WEIGHTED DEGREE CENTRALITY } & \multicolumn{6}{|c|}{ NODE-WEIGHTED EIGEN-VECTOR CENTRALITY } & \multicolumn{6}{|c|}{ NODE-WEIGHTED BETWEENNESS CENTRALITY } & \multicolumn{6}{|c|}{ NODE-WEIGHTED CLOSENESS CENTRALITY } \\
\hline о्山े & तों & 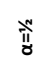 & 突 & خેّ & त्र & त्ञ & तì & तĩ & 离 & خेّ & त्र & 商 & तîं & tĩ & 商 & त्र & స्च̃ & iì & तit & i⿱艹 & तì & خें & त्र & ${ }_{\frac{\pi}{\|}}^{*}$ \\
\hline 1 & $\begin{array}{r}4487 \\
1.4\end{array}$ & $\begin{array}{r}636 \\
09 . \\
9\end{array}$ & $\begin{array}{r}9017 \\
3.7\end{array}$ & $\begin{array}{r}181 \\
213 . \\
3\end{array}$ & $\begin{array}{r}2568 \\
88.7\end{array}$ & $\begin{array}{r}3641 \\
66.5\end{array}$ & $\begin{array}{r}514 \\
95 . \\
5\end{array}$ & $\begin{array}{r}837 \\
76 . \\
9\end{array}$ & $\begin{array}{r}136 \\
294 \\
.6\end{array}$ & $\begin{array}{r}3607 \\
34.7\end{array}$ & $\begin{array}{r}586 \\
870 \\
.9\end{array}$ & $\begin{array}{r}954 \\
766 \\
.6\end{array}$ & $\begin{array}{r}392 \\
65 . \\
4\end{array}$ & $\begin{array}{r}487 \\
08 . \\
6\end{array}$ & $\begin{array}{r}6042 \\
2.8\end{array}$ & $\begin{array}{r}929 \\
80.5\end{array}$ & $\begin{array}{r}11534 \\
2.0\end{array}$ & $\begin{array}{r}14308 \\
1.3\end{array}$ & $\begin{array}{r}194 \\
79.8\end{array}$ & $\begin{array}{r}119 \\
88.2\end{array}$ & $\begin{array}{r}737 \\
7.7\end{array}$ & 2794.2 & $\begin{array}{r}171 \\
9.6\end{array}$ & $\begin{array}{r}105 \\
8.3\end{array}$ \\
\hline 3 & $\begin{array}{r}9768 \\
0.6\end{array}$ & $\begin{array}{r}141 \\
956 \\
.1\end{array}$ & $\begin{array}{r}2063 \\
00.4\end{array}$ & $\begin{array}{r}435 \\
704 . \\
2\end{array}$ & $\begin{array}{r}6331 \\
95.2\end{array}$ & $\begin{array}{r}9202 \\
02.8\end{array}$ & $\begin{array}{r}111 \\
193 \\
.7\end{array}$ & $\begin{array}{r}183 \\
949 \\
.4\end{array}$ & $\begin{array}{r}304 \\
310 \\
.2\end{array}$ & $\begin{array}{r}8328 \\
22.6 \\
\end{array}$ & $\begin{array}{r}137 \\
775 \\
0.4\end{array}$ & $\begin{array}{r}227 \\
923 \\
2.3\end{array}$ & $\begin{array}{r}867 \\
12 . \\
6\end{array}$ & $\begin{array}{r}111 \\
867 \\
.1\end{array}$ & $\begin{array}{r}1443 \\
18.5\end{array}$ & $\begin{array}{r}240 \\
193 . \\
9\end{array}$ & $\begin{array}{r}30987 \\
1.7\end{array}$ & $\begin{array}{r}39976 \\
2.4\end{array}$ & $\begin{array}{r}424 \\
68.9\end{array}$ & $\begin{array}{r}268 \\
33.7\end{array}$ & $\begin{array}{r}169 \\
54.7\end{array}$ & 6768.7 & $\begin{array}{r}427 \\
6.8\end{array}$ & $\begin{array}{l}270 \\
2.2\end{array}$ \\
\hline 4 & $\begin{array}{r}6535 \\
9.7\end{array}$ & $\begin{array}{r}103 \\
170 \\
.8\end{array}$ & $\begin{array}{r}1628 \\
55.8\end{array}$ & $\begin{array}{r}405 \\
785 . \\
6\end{array}$ & $\begin{array}{r}6405 \\
35.6\end{array}$ & $\begin{array}{l}1011 \\
090.4\end{array}$ & $\begin{array}{r}736 \\
21 . \\
3\end{array}$ & $\begin{array}{r}130 \\
901 \\
.3\end{array}$ & $\begin{array}{r}232 \\
747 \\
.0\end{array}$ & $\begin{array}{r}7358 \\
08.2\end{array}$ & $\begin{array}{r}130 \\
829 \\
2.6\end{array}$ & $\begin{array}{c}232 \\
618 \\
9.8\end{array}$ & $\begin{array}{r}593 \\
36 . \\
6\end{array}$ & $\begin{array}{r}850 \\
32 . \\
0\end{array}$ & $\begin{array}{r}1218 \\
54.5\end{array}$ & $\begin{array}{r}250 \\
242 . \\
3\end{array}$ & $\begin{array}{r}35860 \\
8.2\end{array}$ & $\begin{array}{r}51390 \\
1.2\end{array}$ & $\begin{array}{r}277 \\
18.2\end{array}$ & $\begin{array}{r}185 \\
55.2\end{array}$ & $\begin{array}{l}124 \\
21.3\end{array}$ & 5566.4 & $\begin{array}{r}372 \\
6.3\end{array}$ & $\begin{array}{r}249 \\
4.4\end{array}$ \\
\hline 5 & $\begin{array}{r}1267 \\
71.6\end{array}$ & $\begin{array}{r}201 \\
788 \\
.8\end{array}$ & $\begin{array}{r}3211 \\
97.7\end{array}$ & $\begin{array}{r}813 \\
810 . \\
0\end{array}$ & $\begin{array}{r}1295 \\
383 . \\
4\end{array}$ & $\begin{array}{r}2061 \\
928.6\end{array}$ & $\begin{array}{r}145 \\
086 \\
.8\end{array}$ & $\begin{array}{r}264 \\
307 \\
.3\end{array}$ & $\begin{array}{r}481 \\
493 \\
.6\end{array}$ & $\begin{array}{c}1597 \\
913.3\end{array}$ & $\begin{array}{c}291 \\
094 \\
8.7\end{array}$ & $\begin{array}{r}530 \\
293 \\
0.0\end{array}$ & $\begin{array}{r}103 \\
780 \\
.1\end{array}$ & $\begin{array}{r}135 \\
232 \\
.6\end{array}$ & $\begin{array}{r}1762 \\
17.4\end{array}$ & $\begin{array}{r}299 \\
215 . \\
2\end{array}$ & $\begin{array}{r}38989 \\
8.1\end{array}$ & $\begin{array}{r}50806 \\
4.2\end{array}$ & $\begin{array}{r}550 \\
37.5\end{array}$ & $\begin{array}{r}380 \\
33.9\end{array}$ & $\begin{array}{r}262 \\
83.4\end{array}$ & $\begin{array}{r}12551 . \\
8\end{array}$ & $\begin{array}{r}867 \\
4.0 \\
\end{array}$ & $\begin{array}{r}599 \\
4.2 \\
\end{array}$ \\
\hline $\begin{array}{l}1 \\
1\end{array}$ & $\begin{array}{r}3654 \\
8.1\end{array}$ & $\begin{array}{r}587 \\
14 . \\
3 \\
\end{array}$ & $\begin{array}{r}9432 \\
4.3 \\
\end{array}$ & $\begin{array}{r}243 \\
434 . \\
6 \\
\end{array}$ & $\begin{array}{r}3910 \\
76.5 \\
\end{array}$ & $\begin{array}{r}6282 \\
62.6 \\
\end{array}$ & $\begin{array}{r}415 \\
43 . \\
2 \\
\end{array}$ & $\begin{array}{r}758 \\
60 . \\
3 \\
\end{array}$ & $\begin{array}{r}138 \\
525 \\
.5 \\
\end{array}$ & $\begin{array}{r}4619 \\
12.0 \\
\end{array}$ & $\begin{array}{r}843 \\
478 \\
.6 \\
\end{array}$ & $\begin{array}{r}154 \\
024 \\
1.6\end{array}$ & $\begin{array}{r}317 \\
20 . \\
4\end{array}$ & $\begin{array}{r}442 \\
27 . \\
5 \\
\end{array}$ & $\begin{array}{r}6166 \\
6.1 \\
\end{array}$ & $\begin{array}{r}119 \\
882 . \\
1 \\
\end{array}$ & $\begin{array}{r}16715 \\
0.6 \\
\end{array}$ & $\begin{array}{r}23305 \\
6.8 \\
\end{array}$ & $\begin{array}{r}156 \\
91.6 \\
\end{array}$ & $\begin{array}{r}108 \\
23.0 \\
\end{array}$ & $\begin{array}{r}746 \\
5.0 \\
\end{array}$ & 3551.3 & $\begin{array}{r}244 \\
9.5 \\
\end{array}$ & $\begin{array}{r}168 \\
9.5 \\
\end{array}$ \\
\hline $\begin{array}{l}1 \\
3 \\
\end{array}$ & $\begin{array}{r}3896 . \\
4 \\
\end{array}$ & $\begin{array}{r}554 \\
4.0 \\
\end{array}$ & $\begin{array}{r}7888 . \\
5 \\
\end{array}$ & $\begin{array}{r}159 \\
70.6 \\
\end{array}$ & $\begin{array}{r}2272 \\
4.1 \\
\end{array}$ & $\begin{array}{r}3233 \\
3.5 \\
\end{array}$ & $\begin{array}{r}446 \\
5.1 \\
\end{array}$ & $\begin{array}{r}728 \\
0.7 \\
\end{array}$ & $\begin{array}{r}118 \\
71 . \\
6 \\
\end{array}$ & $\begin{array}{r}3156 \\
3.2 \\
\end{array}$ & $\begin{array}{r}514 \\
65 . \\
8 \\
\end{array}$ & $\begin{array}{r}839 \\
18 . \\
1 \\
\end{array}$ & $\begin{array}{r}329 \\
0.5 \\
\end{array}$ & $\begin{array}{r}395 \\
3.8 \\
\end{array}$ & $\begin{array}{r}4750 . \\
9 \\
\end{array}$ & $\begin{array}{r}685 \\
9.5 \\
\end{array}$ & 8242.4 & 9904.0 & $\begin{array}{r}169 \\
0.3 \\
\end{array}$ & $\begin{array}{r}104 \\
3.4 \\
\end{array}$ & $\begin{array}{r}644 . \\
1 \\
\end{array}$ & 245.4 & $\begin{array}{r}151 . \\
5 \\
\end{array}$ & 93.5 \\
\hline $\begin{array}{l}1 \\
5\end{array}$ & $\begin{array}{r}3199 . \\
0\end{array}$ & $\begin{array}{r}461 \\
7.4 \\
\end{array}$ & $\begin{array}{r}6664 . \\
5\end{array}$ & $\begin{array}{r}138 \\
83.9\end{array}$ & $\begin{array}{r}2003 \\
9.3\end{array}$ & $\begin{array}{r}2892 \\
3.8\end{array}$ & $\begin{array}{r}366 \\
2.5\end{array}$ & $\begin{array}{r}605 \\
2.2\end{array}$ & $\begin{array}{r}100 \\
01 . \\
1\end{array}$ & $\begin{array}{r}2730 \\
9.7\end{array}$ & $\begin{array}{r}451 \\
28 . \\
5\end{array}$ & $\begin{array}{r}745 \\
73 . \\
5\end{array}$ & $\begin{array}{r}274 \\
2.1 \\
\end{array}$ & $\begin{array}{r}339 \\
2.4 \\
\end{array}$ & $\begin{array}{r}4196 . \\
9\end{array}$ & $\begin{array}{r}642 \\
3.7 \\
\end{array}$ & 7947.2 & 9832.0 & $\begin{array}{r}142 \\
1.0 \\
\end{array}$ & $\begin{array}{r}911 . \\
0\end{array}$ & $\begin{array}{r}584 . \\
1\end{array}$ & 240.1 & $\begin{array}{r}153 . \\
9\end{array}$ & 98.7 \\
\hline
\end{tabular}

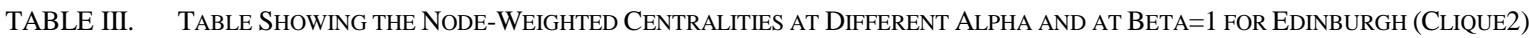

\begin{tabular}{|c|c|c|c|c|c|c|c|c|c|c|c|c|c|c|c|c|c|c|c|c|c|c|c|c|}
\hline \multirow[b]{2}{*}{$\begin{array}{l}\text { 㟔 } \\
\text { Oे }\end{array}$} & \multicolumn{6}{|c|}{$\begin{array}{l}\text { NODE-WEIGHTED DEGREE } \\
\text { CENTRALITY }\end{array}$} & \multicolumn{6}{|c|}{$\begin{array}{l}\text { NODE-WEIGHTED EIGEN-VECTOR } \\
\text { CENTRALITY }\end{array}$} & \multicolumn{6}{|c|}{$\begin{array}{l}\text { NODE-WEIGHTED BETWEENNESS } \\
\text { CENTRALITY }\end{array}$} & \multicolumn{6}{|c|}{$\begin{array}{c}\text { NODE-WEIGHTED } \\
\text { CLOSENESS CENTRALITY }\end{array}$} \\
\hline & iो & iो & î̃ & సٓ̃ & $\frac{\text { त्र }}{\text { II }}$ & 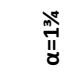 & 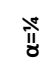 & $\frac{0}{8}$ & III & गें & $\frac{1}{11}$ & $\stackrel{\text { m̃ँ }}{\overrightarrow{I I}}$ & 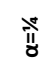 & iो & iIt & $\frac{\overrightarrow{0}}{\not 1}$ & त्र & 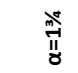 & iì & $\frac{\pi}{8}$ & î̀ & $\frac{\pi}{7}$ & $\frac{\text { â }}{\text { II }}$ & $\prod_{0}^{m}$ \\
\hline 22 & $\begin{array}{r}441 \\
646 . \\
1 \\
\end{array}$ & $\begin{array}{r}48542 \\
8.6 \\
\end{array}$ & $\begin{array}{r}533 \\
551 . \\
6 \\
\end{array}$ & $\begin{array}{r}644 \\
582 . \\
4 \\
\end{array}$ & $\begin{array}{r}70848 \\
3.1 \\
\end{array}$ & $\begin{array}{r}77871 \\
8.5 \\
\end{array}$ & $\begin{array}{r}453 \\
097 . \\
5 \\
\end{array}$ & $\begin{array}{r}510 \\
928 . \\
4\end{array}$ & $\begin{array}{r}576 \\
140 . \\
5 \\
\end{array}$ & $\begin{array}{r}73259 \\
6.9 \\
\end{array}$ & $\begin{array}{r}826 \\
101 . \\
5 \\
\end{array}$ & $\begin{array}{r}931 \\
540 . \\
6\end{array}$ & $\begin{array}{r}303 \\
108 . \\
9\end{array}$ & $\begin{array}{r}228 \\
651 . \\
4\end{array}$ & $\begin{array}{r}172 \\
484 . \\
2\end{array}$ & $\begin{array}{r}98152 . \\
2\end{array}$ & $\begin{array}{r}74041 \\
.5\end{array}$ & $\begin{array}{r}55853 . \\
5\end{array}$ & $\begin{array}{r}203 \\
579 . \\
3\end{array}$ & $\begin{array}{r}10 \\
31 \\
43 \\
.9\end{array}$ & $\begin{array}{c}52 \\
25 \\
8 . \\
1\end{array}$ & $\begin{array}{r}13 \\
41 \\
4 . \\
5\end{array}$ & $\begin{array}{r}67 \\
96 \\
.5\end{array}$ & $\begin{array}{r}34 \\
43 \\
.5\end{array}$ \\
\hline 23 & $\begin{array}{r}322 \\
32.5 \\
\end{array}$ & $\begin{array}{r}30416 . \\
3 \\
\end{array}$ & $\begin{array}{r}287 \\
02.4 \\
\end{array}$ & $\begin{array}{r}255 \\
58.9 \\
\end{array}$ & $\begin{array}{r}24118 . \\
7 \\
\end{array}$ & $\begin{array}{r}22759 . \\
7\end{array}$ & $\begin{array}{r}338 \\
70.3 \\
\end{array}$ & $\begin{array}{r}335 \\
85.7 \\
\end{array}$ & $\begin{array}{r}333 \\
03.6 \\
\end{array}$ & $\begin{array}{r}32746 . \\
4\end{array}$ & $\begin{array}{r}324 \\
71.3 \\
\end{array}$ & $\begin{array}{r}321 \\
98.5 \\
\end{array}$ & $\begin{array}{r}206 \\
34.5 \\
\end{array}$ & $\begin{array}{r}124 \\
65.4 \\
\end{array}$ & $\begin{array}{r}753 \\
0.4 \\
\end{array}$ & 2748.1 & $\begin{array}{r}1660 . \\
2\end{array}$ & 1002.9 & $\begin{array}{r}152 \\
61.9\end{array}$ & $\begin{array}{r}68 \\
19 \\
.2\end{array}$ & $\begin{array}{r}30 \\
46 \\
.9\end{array}$ & $\begin{array}{c}60 \\
8 . \\
3\end{array}$ & $\begin{array}{c}27 \\
1 . \\
8\end{array}$ & $\begin{array}{r}12 \\
1 . \\
4\end{array}$ \\
\hline 24 & $\begin{array}{r}612 \\
98.6 \\
\end{array}$ & $\begin{array}{r}65972 . \\
5 \\
\end{array}$ & $\begin{array}{r}710 \\
02.7 \\
\end{array}$ & $\begin{array}{r}822 \\
43.2 \\
\end{array}$ & $\begin{array}{r}88514 . \\
1 \\
\end{array}$ & $\begin{array}{r}95263 . \\
1 \\
\end{array}$ & $\begin{array}{r}631 \\
65.7 \\
\end{array}$ & $\begin{array}{r}700 \\
52.7 \\
\end{array}$ & $\begin{array}{r}776 \\
90.6 \\
\end{array}$ & $\begin{array}{r}95555 . \\
4 \\
\end{array}$ & $\begin{array}{r}105 \\
973 . \\
8 \\
\end{array}$ & $\begin{array}{r}117 \\
528 . \\
2\end{array}$ & $\begin{array}{r}420 \\
51.1 \\
\end{array}$ & $\begin{array}{r}310 \\
46.7 \\
\end{array}$ & $\begin{array}{r}229 \\
22.1 \\
\end{array}$ & $\begin{array}{r}12494 . \\
9 \\
\end{array}$ & $\begin{array}{r}9225 . \\
1 \\
\end{array}$ & 6811.0 & $\begin{array}{r}280 \\
01.3 \\
\end{array}$ & $\begin{array}{r}13 \\
76 \\
6 . \\
3 \\
\end{array}$ & $\begin{array}{r}67 \\
68 \\
.0 \\
\end{array}$ & $\begin{array}{r}16 \\
35 \\
.8 \\
\end{array}$ & $\begin{array}{r}80 \\
4 . \\
2 \\
\end{array}$ & $\begin{array}{r}39 \\
5 . \\
4\end{array}$ \\
\hline 25 & $\begin{array}{r}742 \\
06.1 \\
\end{array}$ & $\begin{array}{r}69365 . \\
2 \\
\end{array}$ & $\begin{array}{r}648 \\
40.2 \\
\end{array}$ & $\begin{array}{r}566 \\
56.4 \\
\end{array}$ & $\begin{array}{r}52960 . \\
4\end{array}$ & $\begin{array}{r}49505 . \\
5\end{array}$ & $\begin{array}{r}785 \\
87.1 \\
\end{array}$ & $\begin{array}{r}777 \\
97.5 \\
\end{array}$ & $\begin{array}{r}770 \\
15.8 \\
\end{array}$ & $\begin{array}{r}75475 . \\
8\end{array}$ & $\begin{array}{r}747 \\
17.4 \\
\end{array}$ & $\begin{array}{r}739 \\
66.6 \\
\end{array}$ & $\begin{array}{r}468 \\
33.4\end{array}$ & $\begin{array}{r}276 \\
29.6\end{array}$ & $\begin{array}{r}163 \\
00.2\end{array}$ & 5673.2 & $\begin{array}{r}3346 . \\
9 \\
\end{array}$ & 1974.5 & $\begin{array}{r}364 \\
35.0 \\
\end{array}$ & $\begin{array}{r}16 \\
72 \\
2 . \\
4\end{array}$ & $\begin{array}{r}76 \\
75 \\
.0 \\
\end{array}$ & $\begin{array}{r}16 \\
16 \\
.8 \\
\end{array}$ & $\begin{array}{c}74 \\
2 . \\
0 \\
\end{array}$ & $\begin{array}{r}34 \\
0 . \\
6 \\
\end{array}$ \\
\hline 30 & $\begin{array}{r}144 \\
722 . \\
5\end{array}$ & $\begin{array}{r}13898 \\
8.2 \\
\end{array}$ & $\begin{array}{r}133 \\
481 . \\
0 \\
\end{array}$ & $\begin{array}{r}123 \\
112 . \\
8 \\
\end{array}$ & $\begin{array}{r}11823 \\
4.7 \\
\end{array}$ & $\begin{array}{r}11354 \\
9.9 \\
\end{array}$ & $\begin{array}{r}152 \\
807 . \\
8 \\
\end{array}$ & $\begin{array}{r}154 \\
952 . \\
0\end{array}$ & $\begin{array}{r}157 \\
126 . \\
2 \\
\end{array}$ & $\begin{array}{r}16156 \\
6.5 \\
\end{array}$ & $\begin{array}{r}163 \\
833 . \\
5 \\
\end{array}$ & $\begin{array}{r}166 \\
132 . \\
3\end{array}$ & $\begin{array}{r}921 \\
32.9 \\
\end{array}$ & $\begin{array}{r}563 \\
29.4 \\
\end{array}$ & $\begin{array}{r}344 \\
39.4 \\
\end{array}$ & $\begin{array}{r}12873 . \\
5 \\
\end{array}$ & $\begin{array}{r}7870 . \\
7 \\
\end{array}$ & 4812.1 & $\begin{array}{r}694 \\
73.0 \\
\end{array}$ & $\begin{array}{c}32 \\
02 \\
8 . \\
6 \\
\end{array}$ & $\begin{array}{c}14 \\
76 \\
5 . \\
9 \\
\end{array}$ & $\begin{array}{r}31 \\
38 \\
.4 \\
\end{array}$ & $\begin{array}{r}14 \\
46 \\
.9 \\
\end{array}$ & $\begin{array}{r}66 \\
7 . \\
0 \\
\end{array}$ \\
\hline 31 & $\begin{array}{r}318 \\
409 . \\
5 \\
\end{array}$ & $\begin{array}{r}33837 \\
2.0 \\
\end{array}$ & $\begin{array}{r}359 \\
586 . \\
2 \\
\end{array}$ & $\begin{array}{r}406 \\
087 . \\
9 \\
\end{array}$ & $\begin{array}{r}43154 \\
7.4 \\
\end{array}$ & $\begin{array}{r}45860 \\
3.1 \\
\end{array}$ & $\begin{array}{r}328 \\
540 . \\
4 \\
\end{array}$ & $\begin{array}{r}360 \\
246 . \\
8 \\
\end{array}$ & $\begin{array}{r}395 \\
013 . \\
1 \\
\end{array}$ & $\begin{array}{r}47493 \\
5.1 \\
\end{array}$ & $\begin{array}{r}520 \\
769 . \\
5 \\
\end{array}$ & $\begin{array}{r}571 \\
027 . \\
4 \\
\end{array}$ & $\begin{array}{r}215 \\
012 . \\
3 \\
\end{array}$ & $\begin{array}{r}154 \\
294 . \\
1 \\
\end{array}$ & $\begin{array}{r}110 \\
722 . \\
3 \\
\end{array}$ & $\begin{array}{r}57017 . \\
4\end{array}$ & $\begin{array}{r}40916 \\
.0 \\
\end{array}$ & $\begin{array}{r}29361 . \\
6 \\
\end{array}$ & $\begin{array}{r}143 \\
261 . \\
1 \\
\end{array}$ & $\begin{array}{r}68 \\
49 \\
8 . \\
2\end{array}$ & $\begin{array}{r}32 \\
75 \\
1 . \\
4\end{array}$ & $\begin{array}{r}74 \\
87 \\
.4 \\
\end{array}$ & $\begin{array}{r}35 \\
80 \\
.0 \\
\end{array}$ & $\begin{array}{r}17 \\
11 \\
.7 \\
\end{array}$ \\
\hline 32 & $\begin{array}{r}290 \\
386 . \\
7\end{array}$ & $\begin{array}{r}33044 \\
2.0 \\
\end{array}$ & $\begin{array}{r}376 \\
022 . \\
5\end{array}$ & $\begin{array}{r}486 \\
912 . \\
5\end{array}$ & $\begin{array}{r}55407 \\
6.2 \\
\end{array}$ & $\begin{array}{r}63050 \\
4.3 \\
\end{array}$ & $\begin{array}{r}297 \\
308 . \\
6 \\
\end{array}$ & $\begin{array}{r}346 \\
383 . \\
0 \\
\end{array}$ & $\begin{array}{r}403 \\
557 . \\
8 \\
\end{array}$ & $\begin{array}{r}54777 \\
7.5 \\
\end{array}$ & $\begin{array}{r}638 \\
195 . \\
0 \\
\end{array}$ & $\begin{array}{r}743 \\
537 . \\
0 \\
\end{array}$ & $\begin{array}{r}198 \\
860 . \\
2 \\
\end{array}$ & $\begin{array}{r}154 \\
966 . \\
4 \\
\end{array}$ & $\begin{array}{r}120 \\
761 . \\
2 \\
\end{array}$ & $\begin{array}{r}73334 . \\
2 \\
\end{array}$ & $\begin{array}{r}57147 \\
.4 \\
\end{array}$ & $\begin{array}{r}44533 . \\
4 \\
\end{array}$ & $\begin{array}{r}132 \\
057 . \\
8 \\
\end{array}$ & $\begin{array}{r}68 \\
33 \\
9 . \\
2 \\
\end{array}$ & $\begin{array}{r}35 \\
36 \\
5 . \\
2 \\
\end{array}$ & $\begin{array}{r}94 \\
70 \\
.8 \\
\end{array}$ & $\begin{array}{l}49 \\
01 \\
.1 \\
\end{array}$ & $\begin{array}{r}25 \\
36 \\
.3 \\
\end{array}$ \\
\hline 33 & $\begin{array}{r}157 \\
564 . \\
6 \\
\end{array}$ & $\begin{array}{r}15762 \\
9.0 \\
\end{array}$ & $\begin{array}{r}157 \\
693 . \\
5 \\
\end{array}$ & $\begin{array}{r}157 \\
822 . \\
4\end{array}$ & $\begin{array}{r}15788 \\
6.9 \\
\end{array}$ & $\begin{array}{r}15795 \\
1.5 \\
\end{array}$ & $\begin{array}{r}165 \\
476 . \\
9 \\
\end{array}$ & $\begin{array}{r}173 \\
857 . \\
7 \\
\end{array}$ & $\begin{array}{r}182 \\
662 . \\
9\end{array}$ & $\begin{array}{r}20163 \\
3.7 \\
\end{array}$ & $\begin{array}{r}211 \\
845 . \\
6 \\
\end{array}$ & $\begin{array}{r}222 \\
574 . \\
7 \\
\end{array}$ & $\begin{array}{r}102 \\
367 . \\
7\end{array}$ & $\begin{array}{r}665 \\
34.2 \\
\end{array}$ & $\begin{array}{r}432 \\
44.1 \\
\end{array}$ & $\begin{array}{r}18268 . \\
0 \\
\end{array}$ & $\begin{array}{r}11873 \\
.3 \\
\end{array}$ & 7717.1 & $\begin{array}{r}746 \\
47.8 \\
\end{array}$ & $\begin{array}{c}35 \\
37 \\
9 . \\
6 \\
\end{array}$ & $\begin{array}{r}16 \\
76 \\
8 . \\
3 \\
\end{array}$ & $\begin{array}{r}37 \\
66 \\
.7 \\
\end{array}$ & $\begin{array}{r}17 \\
85 \\
.2 \\
\end{array}$ & $\begin{array}{r}84 \\
6 . \\
1 \\
\end{array}$ \\
\hline 35 & $\begin{array}{r}322 \\
9.5 \\
\end{array}$ & 3048.7 & $\begin{array}{r}287 \\
8.0 \\
\end{array}$ & $\begin{array}{r}256 \\
4.8 \\
\end{array}$ & 2421.2 & 2285.7 & $\begin{array}{r}341 \\
2.6 \\
\end{array}$ & $\begin{array}{r}340 \\
4.2 \\
\end{array}$ & $\begin{array}{r}339 \\
5.9 \\
\end{array}$ & 3379.3 & $\begin{array}{r}337 \\
1.0 \\
\end{array}$ & $\begin{array}{r}336 \\
2.7 \\
\end{array}$ & $\begin{array}{r}200 \\
6.6 \\
\end{array}$ & $\begin{array}{r}117 \\
7.0 \\
\end{array}$ & $\begin{array}{r}690 . \\
3 \\
\end{array}$ & 237.5 & 139.3 & 81.7 & $\begin{array}{r}157 \\
5.7 \\
\end{array}$ & $\begin{array}{r}72 \\
5 . \\
8 \\
\end{array}$ & $\begin{array}{r}33 \\
4 . \\
3\end{array}$ & $\begin{array}{r}70 \\
.9 \\
\end{array}$ & $\begin{array}{r}32 \\
.7 \\
\end{array}$ & $\begin{array}{r}15 \\
.0 \\
\end{array}$ \\
\hline 36 & $\begin{array}{r}199 \\
42.1 \\
\end{array}$ & $\begin{array}{r}18730 . \\
5 \\
\end{array}$ & $\begin{array}{r}175 \\
92.4 \\
\end{array}$ & $\begin{array}{r}155 \\
19.5 \\
\end{array}$ & $\begin{array}{r}14576 . \\
5 \\
\end{array}$ & $\begin{array}{r}13690 . \\
9 \\
\end{array}$ & $\begin{array}{r}211 \\
28.7 \\
\end{array}$ & $\begin{array}{r}210 \\
25.6 \\
\end{array}$ & $\begin{array}{r}209 \\
23.1 \\
\end{array}$ & $\begin{array}{r}20719 . \\
5 \\
\end{array}$ & $\begin{array}{r}206 \\
18.5 \\
\end{array}$ & $\begin{array}{r}205 \\
18.0 \\
\end{array}$ & $\begin{array}{r}124 \\
34.6 \\
\end{array}$ & $\begin{array}{r}728 \\
2.3 \\
\end{array}$ & $\begin{array}{r}426 \\
4.8 \\
\end{array}$ & 1462.8 & 856.7 & 501.7 & $\begin{array}{r}973 \\
4.5 \\
\end{array}$ & $\begin{array}{r}44 \\
63 \\
.0 \\
\end{array}$ & $\begin{array}{r}20 \\
46 \\
.2 \\
\end{array}$ & $\begin{array}{r}43 \\
0 . \\
1 \\
\end{array}$ & $\begin{array}{r}19 \\
7 . \\
2\end{array}$ & $\begin{array}{r}90 \\
.4 \\
\end{array}$ \\
\hline 37 & $\begin{array}{r}503 \\
6.8 \\
\end{array}$ & 5554.6 & $\begin{array}{r}612 \\
5.7 \\
\end{array}$ & $\begin{array}{r}745 \\
0.1 \\
\end{array}$ & 8216.0 & 9060.8 & $\begin{array}{r}528 \\
5.0 \\
\end{array}$ & $\begin{array}{r}611 \\
5.7 \\
\end{array}$ & $\begin{array}{r}707 \\
6.9 \\
\end{array}$ & 9476.3 & $\begin{array}{r}109 \\
65.7 \\
\end{array}$ & $\begin{array}{r}126 \\
89.2 \\
\end{array}$ & $\begin{array}{r}324 \\
7.1 \\
\end{array}$ & $\begin{array}{r}230 \\
8.6 \\
\end{array}$ & $\begin{array}{r}164 \\
1.3 \\
\end{array}$ & 829.6 & 589.8 & 419.4 & $\begin{array}{r}236 \\
2.2 \\
\end{array}$ & $\begin{array}{r}12 \\
21 \\
.8 \\
\end{array}$ & $\begin{array}{r}63 \\
1 . \\
9 \\
\end{array}$ & $\begin{array}{r}16 \\
9 . \\
1 \\
\end{array}$ & $\begin{array}{r}87 \\
.4 \\
\end{array}$ & $\begin{array}{r}45 \\
.2 \\
\end{array}$ \\
\hline
\end{tabular}




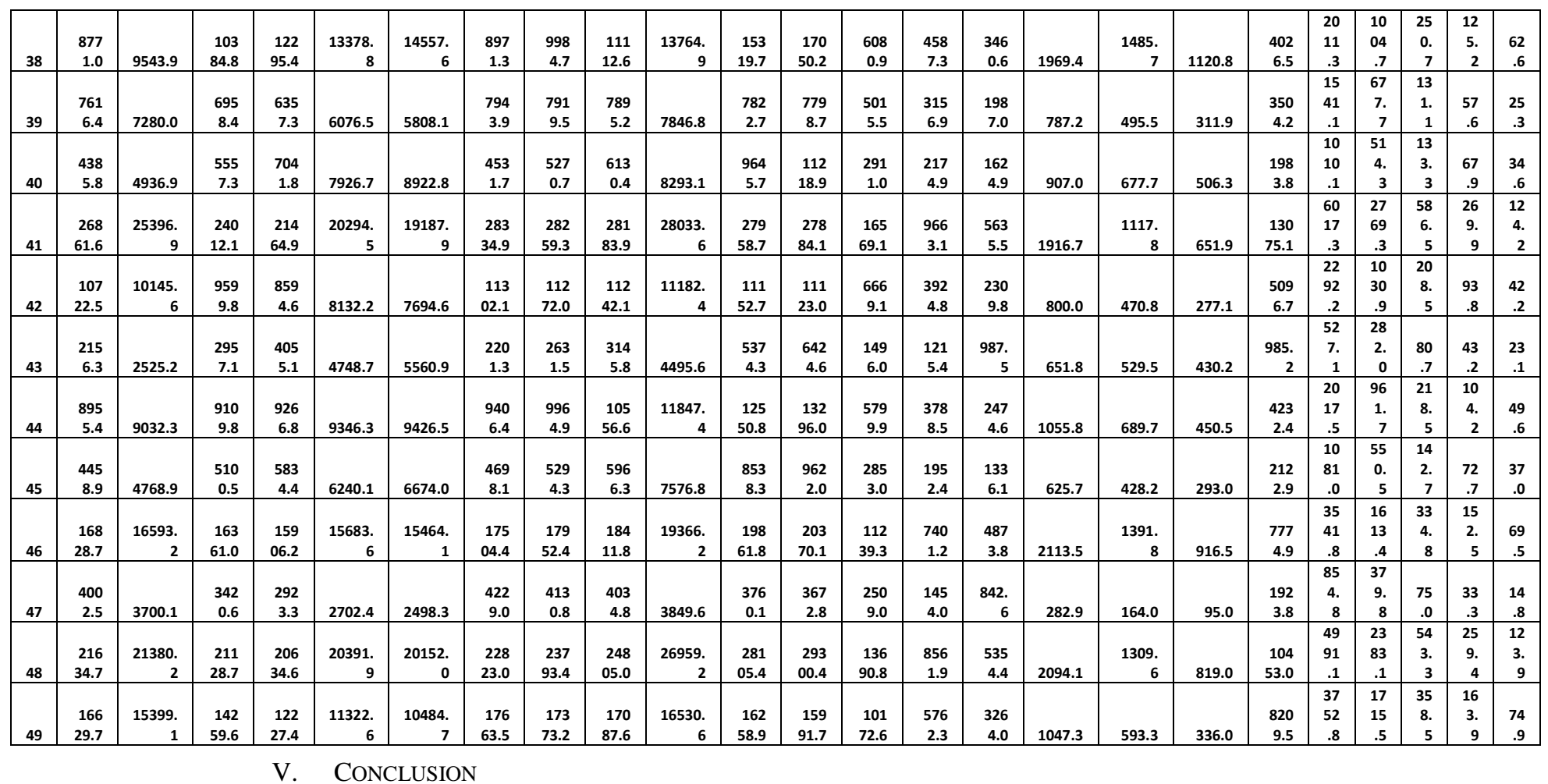

Two case studies were considered in the supply chain management, one considered two cliques with horizontal distance apart (case study of Glasgow clique and Edinburgh cliques in Scotland), while the second case study considered the triangular shaped cliques of (Londonderry, NewtonAbbey and Belfast, in the Northern Ireland).

The results obtained show that the combined weights have an obvious effect on the centralities of the nodes considered as evidenced in the case studies of the Supply Chain Management(SCM). The tuning parameters alpha (whose values range between 0.25 and 1.75 ) acts as the bounds for the relative importance of number of ties/weight of ties and the tuning parameter beta (whose values are -1 and +1 ) serves as multiplicative/dividing factors for weights of nodes.

Graphs in the SCM were considered and effects of the combined weights on edges (distance between shops) and weights on nodes (sales value for SCM) were evaluated taking the betweenness, closeness, eigenvector and degree centrality into cognisance. The resulting node-weight modulated centrality was then applied to the sales dataset while introducing an additional tuning parameter $\beta$ thereby making use of two parameters $\alpha$ and $\beta$.

The resulting predictions in both cases were $94.6 \%$ accurate for the Scotland cliques compared with the accuracy of $76.5 \%$ obtained with the Weighted Marking Method while $96.2 \%$ of accuracy was obtained in the case study involving the Northern Ireland with the clique/node-weighted centrality measure.

\section{FUTURE STUDIES}

The links/edges between nodes might not just be road linkage, two attributes might be considered, e.g communication bandwidth within two nodes and physical distances between them could be combined in future.

This model could be further extended to other datasets such as in the area of disease control, whereby the model can be used to detect the most central region where epidemic diseases are proned to spread easily or to find the most vulnerable group in the society to an epidemic disease. Here the node weight could be the preponderance of an infectious disease in a particular node and the edge weight will be the distance apart from of highly infected nodes to other nodes in such a graph.

\section{ACKNOWLEDGEMENT}

We wish to acknowledge Dunnhumby, United Kingdom (www.dunnhumby.com) for providing the TESCO datasets that were used for the purpose of this research.

\section{REFERENCES}

[1] A.A.G. Akanmu, F.Z. Wang \& A.F. Yamoah(2014). Clique Structure and Node-Weighted Centrality Measures for Predicting Distribution Centre Location in the Supply Chain Management", IEEE Technically Co-Sponsored Science and Information Conference Aug.27-29, 2014, London UK.

[2] A. Barrat, M. Barthelemy, R. Pastor-Satorras, \& A. Vespignani (2004). The Architecture of Complex Weighted Networks. Proceedings of the National Academy of Sciences 101(11), 3747-3752. arXiv:condmat/0311416.

[3] M.S. Granovetter. (1973). The Strength of Weak Ties. American Journal of Sociology, University of Chicago Press. pp. 1360-1380.

[4] U. Brandes (2001). A Faster Algorithm for Betweenness Centrality. Journal of Mathematical Sociology 25, 163-177.

[5] M.E.J. Newman (2001). Scientific Collaboration networks. II. Shortest paths, weighted networks, and centrality. The American Physical Society. Physical review E, Volume 64, 016132

[6] T. Opsahl, F. Agneessens \& J. Skvoretz (2010). Node Centrality in Weighted Networks: Generalizing degree and shortest Paths. Social Networks 32(2010) 245-251. Elsevier B.V. 
[7] H. Zhuge \& J. Zhang(2010). Topological Centrality and It's e-Science Applications. Wiley Interscience.

[8] V.V. Thai \& D. Grewal(2005).Selecting the location of Distribution Centre in Logistics Operations: A Conceptual Framework and Case Study. Asia Pacific Journal of Marketing and Logistics, 17(3), 3-24.
[9] B.V. Carolan(2014). Social Network Analysis and Education: Theory, Methods \& Applications.SAGE Publications, USA. pp120

[10] Mapdata(2014). Properties on Map. Accessed 26 December, 2014 from http://www.rightmove.co.uk/commercial-property-to-

let $/$ map.html?locationIdentifier=REGION\%5E815\&insId=1\&radius $=40$. 0 